\title{
Thermography for Characterisation of Deformation Process in Stainless Steels
}

\author{
by B.Venkatraman*, M.Menaka and Baldev Raj**
}

* Indira Gandhi Centre for Atomic Research, Kalpakkam-603102, India, bvenkat@igcar.gov.in

${ }^{\star *}$ President Research-PSG Institutions, PSG College of Technology Campus, Peelamedu, Coimbatore, India, baldev.dr@gmail.com

\begin{abstract}
It is well known that during tensile testing, a part of the mechanical work done on the specimen is transformed into heat energy. The rate of rise and maximum temperature attained depends on the nature of material, its deformation behaviour and test conditions. This paper highlights the application of thermography for characterising various stages of tensile deformation in AISI type 316 stainless steel and predicting the zone of failure in advance. Multi layered perceptron based ANN was used for predicting the temperature based on the experimental data. Temperature prediction especially in work hardening region could be done with errors less than $1.0 \%$.
\end{abstract}

\section{Introduction}

It is well known that when a metal is plastically deformed and subjected to failure during the tensile test, most of the absorbed strain energy is converted into heat. Consequently, the temperature of the metal increases. The ultimate temperature attained by the specimen and the rate of temperature change including the thermal gradients developed, are directly related to the material properties, physical conditions of the test and deformation behaviour of the material. The measurement of this rise in temperature due to deformation induced heating can give valuable information about the nature and severity of the deformation and also help in gaining a better insight of the complex linkages between mechanisms of deformation with material properties. Literature study reveals that though plastic instabilities in materials due to tensile deformation has been studied through modelling and a number of theories have been proposed [38-41], the idea of using temperature as a sensitive indicator did not catch the fancy of many researchers.

To study deformation induced heating, a means of measuring temperature rapidly and accurately is required. In the past, both contact and non-contact techniques have been used for measuring the temperature. However, such measurements gave only point information and errors associated with these measurements were found to be large $( \pm 1 \mathrm{~K})$. The concept of utilizing an infrared sensor to detect and measure the surface temperature variations started only in the late $70 \mathrm{~s}$. Some of the early researchers include Wilburn [1], Huang et al.[2], Sachdev et al.[3] etc. However all these focussed on carbon steel. No detailed investigations have been reported on the use of IR imaging for studying tensile deformation especially on AISI type 316 stainless steels except by the authors [4].

\section{Experimental Approach}

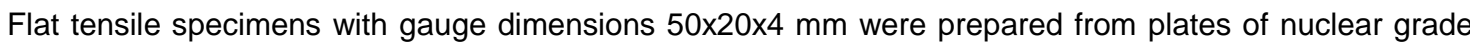
AISI type 316 stainless steel, solution annealed at a temperature of $1323 \mathrm{~K}$ for one hour and water quenched. The specimens are prepared as per the guidelines given in ASTM E 8-95a Standard Test Method for Tension Testing of Metallic Materials. The specimens used for the experimentation were qualified using appropriate NDE techniques to ensure that they meet the dimensional and surface finish requirements. To ensure that they were also free from defects, all the specimens were subjected to high sensitivity radiography. Tensile testing of all the specimens was carried out in a tensile testing machine at ambient temperature with cross head speeds of $1 \mathrm{~mm} / \mathrm{min}, 2 \mathrm{~mm} / \mathrm{min}, 5 \mathrm{~mm} / \mathrm{min}, 10 \mathrm{~mm} / \mathrm{min}, 20 \mathrm{~mm} / \mathrm{min}$ and $50 \mathrm{~mm} / \mathrm{min}$. This represents a broad range spanning from a low strain rate to a comparatively fast one. Thermal images of the specimens during tensile deformation were captured using a focal plane array based system. To overcome the effects of emissivity variations, a thin coat of black paint was applied to the gage length of the specimens. 


\subsection{Results and Discussions}

\subsection{Visualisng stages of tensile deformation through IR Imaging}

A series of systematic experiments was performed to characterise the process of tensile deformation in AISI type 316 stainless steel. The different manifestations of thermal pattern accompanying the plastic deformation of AISI type 316 SS is presented in Fig. 1. This gives an overview of the thermal dissipation as a function of nominal strain for a strain rate of $3.3 \times 10^{-4} \mathrm{~s}^{-1}$. The progressive rise in temperature along the gauge length with increasing strain levels can be clearly visualized from the thermal patterns.

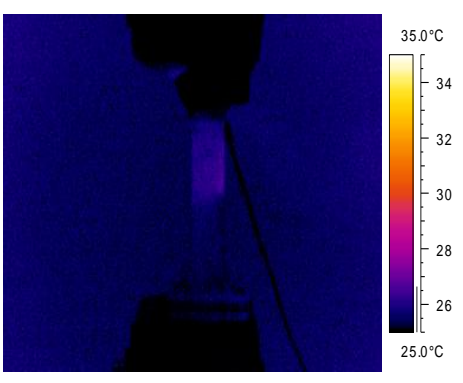

(a)

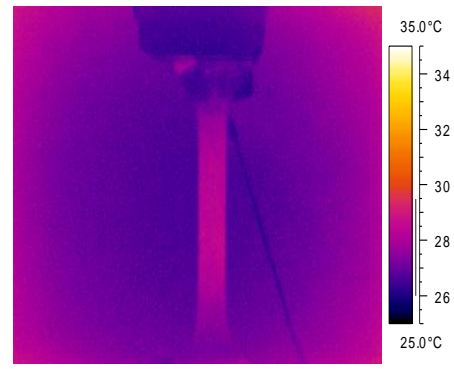

(d)

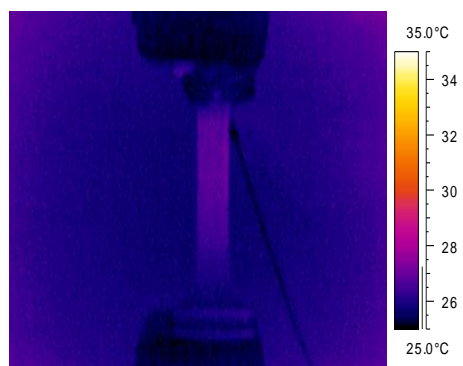

(b)

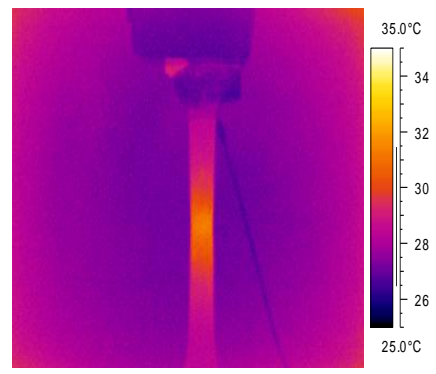

(e)

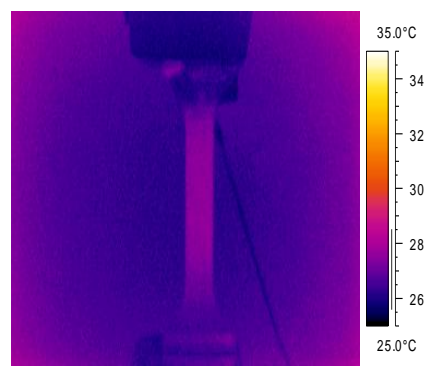

(c)

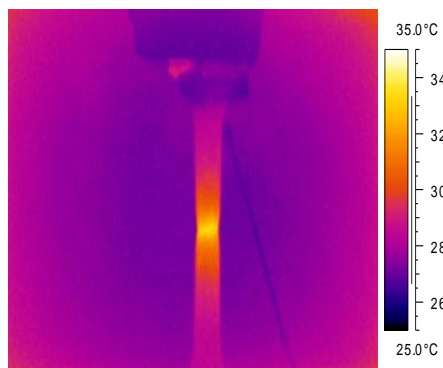

(f)

Fig. 1. Thermal images for typical nominal strain at strain rate of $3.3 \times 10^{-4} \mathrm{~s}^{-1}$.

For quick visualisation of the phenomenon, a graphical plot of maximum temperature observed in the gauge length region versus strain for a strain rate of $3.3 \times 10^{-4} \mathrm{~s}^{-1}$ is presented in Fig. 3.3. The maximum temperature is obtained by thresholding the region using area measurement function, profiling the region and using spot temperature measurement to locate the exact point and obtain the maximum temperature. The curve in general can be divided into three distinct regions namely :

Region I - start of the test to just before yield point.

Region II - from yield point to Ultimate Tensile Strength (UTS) and

Region III - from UTS to fracture. 


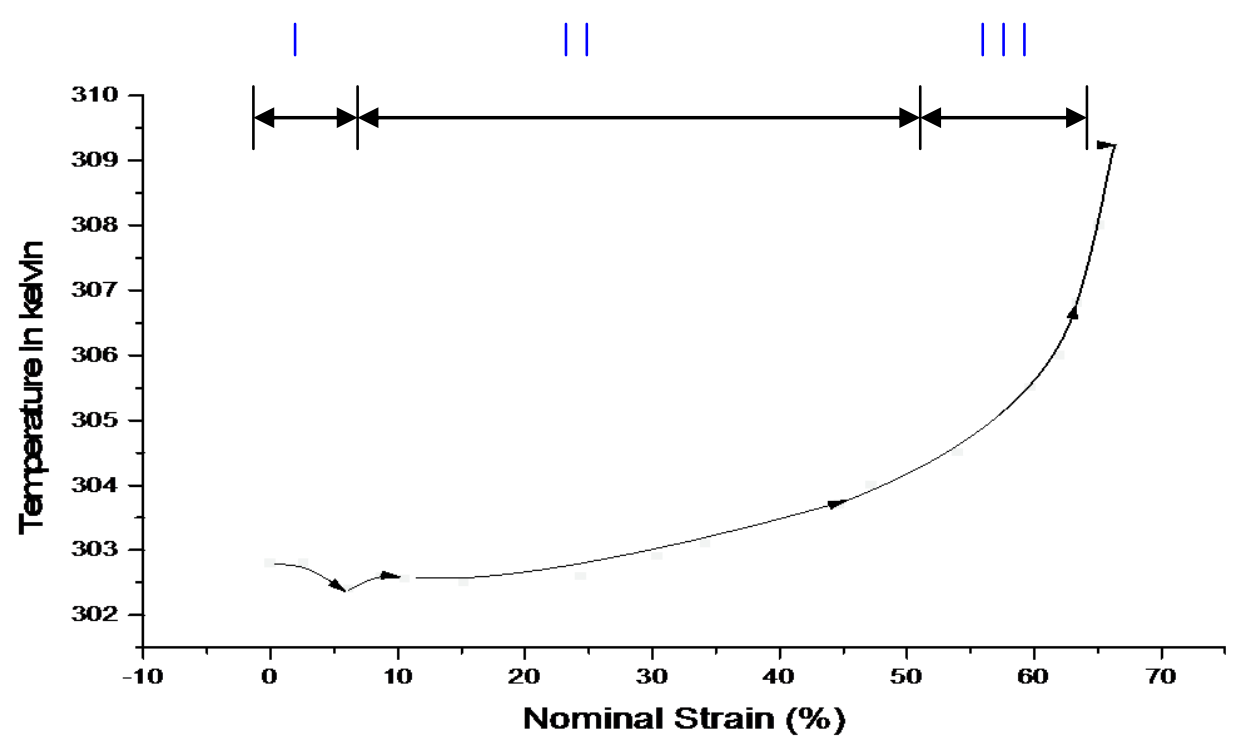

Fig. 2. Variation of nominal strain with temperature for strain rate of $3.3 \times 10^{-4} \mathrm{~s}^{-1}$.

The slight drop in the temperature in the elastic region is attributed to the thermoelastic effect. The initial drop of temperature in the elastic region is followed by a sudden increase in temperature as plastic deformation begins. This inversion of temperature indicates the transition from the elastic to the plastic behaviour of the material. With increasing strain levels, the temperature continues to increase. Region II is the work hardening region characterised by a rise in temperature till UTS point.

Work done during plastic deformation is a thermodynamically irreversible process. A major portion of the total mechanical work done during plastic flow is dissipated as heat which raises the temperature of the material while being deformed. A small but significant percentage of energy, however, is stored in the metal by the formation of lattice defects, mainly dislocations. It has been observed that the amount of heat evolved as a percentage of plastic work expended in the tensile deformation is about $86.5 \%$ for steel, 90.5-92 \% for copper, 92-93\% for polycrystalline aluminium and 95-95.5\% for aluminium single crystal [102]. Heat generated at each point of the specimen is proportional to the energy dissipated $\left(E_{h}\right)$ by the specimen at that point and thus proportional to the level of stress at that point. As the specimen is deformed plastically, work hardening takes place. That is, the stress required to cause additional strain in this region increases with plastic strain. As a result, the heat generated at each point increases with plastic strain. The main mechanism of heat loss during tensile deformation can be considered to be due to conduction. Convection losses can be considered to be minimum due to the small temperature difference between the surrounding and the specimen. Thus, with increasing strain levels, the rate of generation of heat exceeds the rate of conduction. This produces a perceptible rise in the temperature of the specimen which is observed in the IR image.

In general, strain hardening increases the specimen's load carrying capacity. However, at UTS the effect of strain hardening is overcome by the reduction in cross-sectional area of the specimen, resulting in the decrease in its load carrying capacity. Consequently, localized deformation results. This condition is seen as necking of the tensile specimen. The necking of the specimen manifests as a maximum in the engineering stress-strain curve. In the temperature vs strain curve, a large rise in temperature is observed during necking which results in the change in the slope of the strain vs. temperature curve (fig. 2). The reason for this sudden rise in the temperature can be attributed to the mechanism of fracture in ductile materials.

The temperatures were profiled along the gauge length of the specimen. Both spot and line profiles were used to determine the temperature variation and the maximum temperature attained along the gauge length of the specimen. Table-1 gives the maximum temperature observed in the gauge region for nominal strains of $7.3 \%, 15.4 \%, 27 \%, 34.2 \%, 47.7 \%$ and $56.5 \%$ as a function of strain rate. It can be seen from Table-1 that the maximum temperature attained at the centre of the specimen is function of 
strain rate being greater for a strain rate of $1.7 \times 10^{-2} \mathrm{~s}^{-1}$ than $3.33 \times 10^{-4} \mathrm{~s}^{-1}$. The rise in temperature of the specimen with the strain rate for the same values of strain can be explained as follows.

Table 1. Variation of temperature along gauge length for different strains and strain rates

\begin{tabular}{|c|c|c|c|c|c|c|}
\hline \multirow{2}{*}{$\begin{array}{l}\text { Nominal } \\
\text { Strain (\%) } \\
\qquad \begin{array}{l}\text { Strain } \\
\text { rate }\end{array} \rightarrow\end{array}$} & \multicolumn{6}{|c|}{ Temperature in gauge length $(\mathrm{K})$} \\
\hline & $\begin{array}{c}3.3 \times 10^{-4} \\
s^{-1}\end{array}$ & $\begin{array}{c}6.6 \times 10^{-4} \\
\mathrm{~s}^{-1}\end{array}$ & $\begin{array}{c}1.7 \times 10^{-3} \\
\mathrm{~s}^{-1}\end{array}$ & $\begin{array}{c}3.3 \times 10^{-3} \\
\mathrm{~s}^{-1}\end{array}$ & $\begin{array}{c}6.6 \times 10^{-3} \\
\mathrm{~s}^{-1}\end{array}$ & $\begin{array}{c}1.7 \times 10^{-2} \\
\mathrm{~s}^{-1}\end{array}$ \\
\hline 7.3 & 299.9 & 302.6 & 300.3 & 302.7 & 301.3 & 303.9 \\
\hline 15.4 & 300.1 & 302.5 & 301.9 & 303.0 & 302.4 & 304.3 \\
\hline 27.0 & 301.2 & 302.9 & 302.4 & 304.6 & 304.9 & 308.6 \\
\hline 34.2 & 300.8 & 303.1 & 304.6 & 306.5 & 307.2 & 309.4 \\
\hline 47.7 & 301.4 & 304.0 & 306.7 & 310.8 & 314.9 & 320.0 \\
\hline 56.5 & 301.7 & 304.5 & 310.0 & 320.7 & 348.4 & 365.5 \\
\hline
\end{tabular}

As the specimen is deformed plastically, work hardening takes place. It is well known that plastic deformation is due to the movement of large number of dislocations. The rate of plastic deformation i.e., the strain rate $\varepsilon$ is proportional to the Boltzmann's probability factor:

$$
\varepsilon \propto \exp \left(-\mathrm{Q} / \mathrm{k}_{\mathrm{pf}} \mathrm{T}\right)
$$

where $\mathrm{Q}$ is the activation energy for dislocation motion. $\mathrm{Q}$ arises from the activation barrier that a dislocation faces in moving from one minimum energy position to next. The above equation indicates that a dislocation can change its position solely due to thermal fluctuations even without the application of an external stress. Such motion would be random in nature where a dislocation would have an equal probability of moving to the next minimum energy position in any direction. Such motions however would not result in plastic deformation. A certain threshold stress is thus necessary for dislocations to move in a specified direction. If $\tau_{\mathrm{PN}}$ is the stress required to move a dislocation in the absence of thermal energy (at $0 \mathrm{~K}$ ) and $\tau_{\mathrm{app}}$ is the applied stress at $0 \mathrm{~K}$, then

$$
\mathrm{Q}=\left(\tau_{\mathrm{PN}}-\tau_{\mathrm{app}}\right) \gamma
$$

where $\gamma$ is the activation volume. Substituting this in equation (3.9) we have

$$
\dot{\varepsilon} \propto \exp -\left[\left[\frac{\left.\tau_{\mathrm{PN}}-\tau_{\mathrm{app}}\right] \gamma}{\mathrm{kpf}_{\mathrm{pf}} T}\right]\right.
$$

For a given material, $\gamma$ and $\tau$ PN are constant. It can thus be observed from this equation that when an experiment is performed at constant ambient temperature ( $299 \mathrm{~K}$ in the present case), the strain rate is proportional to the applied stress. The higher the strain rate, the higher the stress. The heat generated at each point on the specimen is proportional to the energy dissipated by the specimen at that point and thus proportional to the level of stress at the point. Hence for the same nominal strain, higher strain rate produces a higher energy thus resulting in higher temperature.

An additional reason for the higher temperature at a higher strain rate is the mechanism of thermal conduction. At lower strain rates, heat is generated rather slowly by the deformation process and is dissipated to the surroundings quickly resulting in lower rise in the temperature. But with increasing strain rate, the deformation process tends towards an adiabatic one, i.e., the rate at which the specimen is deformed increases than the rate at which heat is conducted to the surroundings, causing the temperature to rise to a higher value as compared to the temperature attained for the same strain levels but at a lower strain rate value.

\subsection{Early Prediction of the Zone of Failure through IR imaging [5]}

Figs. 3. (a)-(b) show typical plots of the variation of temperature on the specimen along its gauge length as a function of nominal strain for two different strain rates. The point of maximum temperature was determined through thermal profiling for each nominal strain and the graph has been plotted for temperatures on either side of the maximum. 


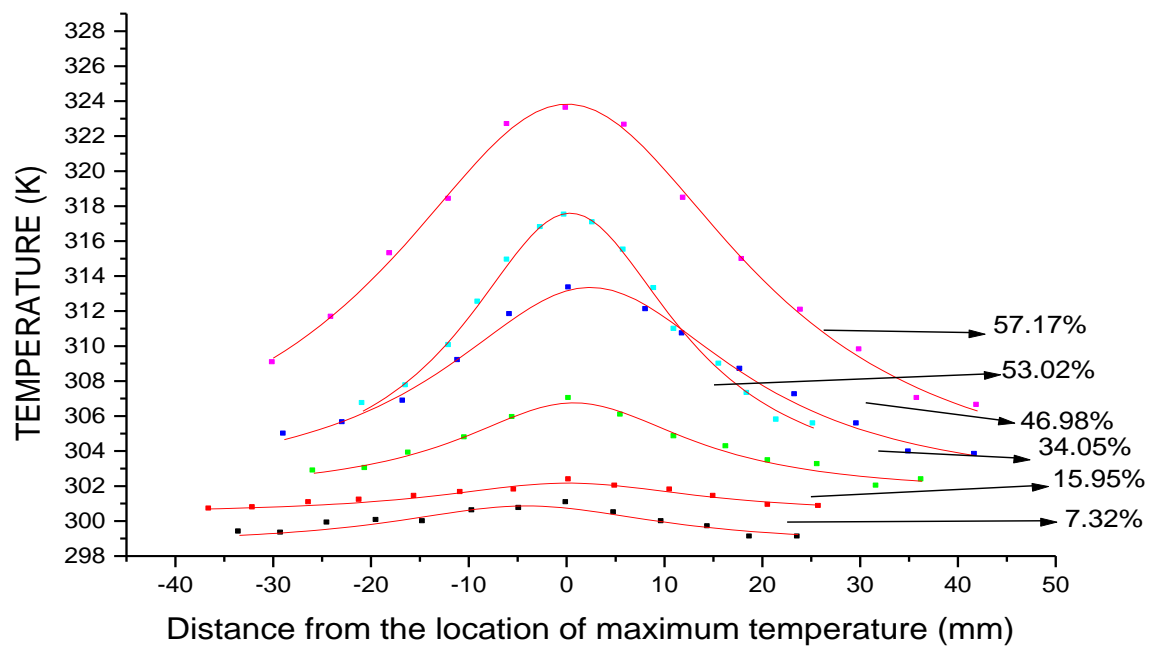

(a) Strain rate of $6.7 \times 10^{-3} \mathrm{~s}^{-1}$

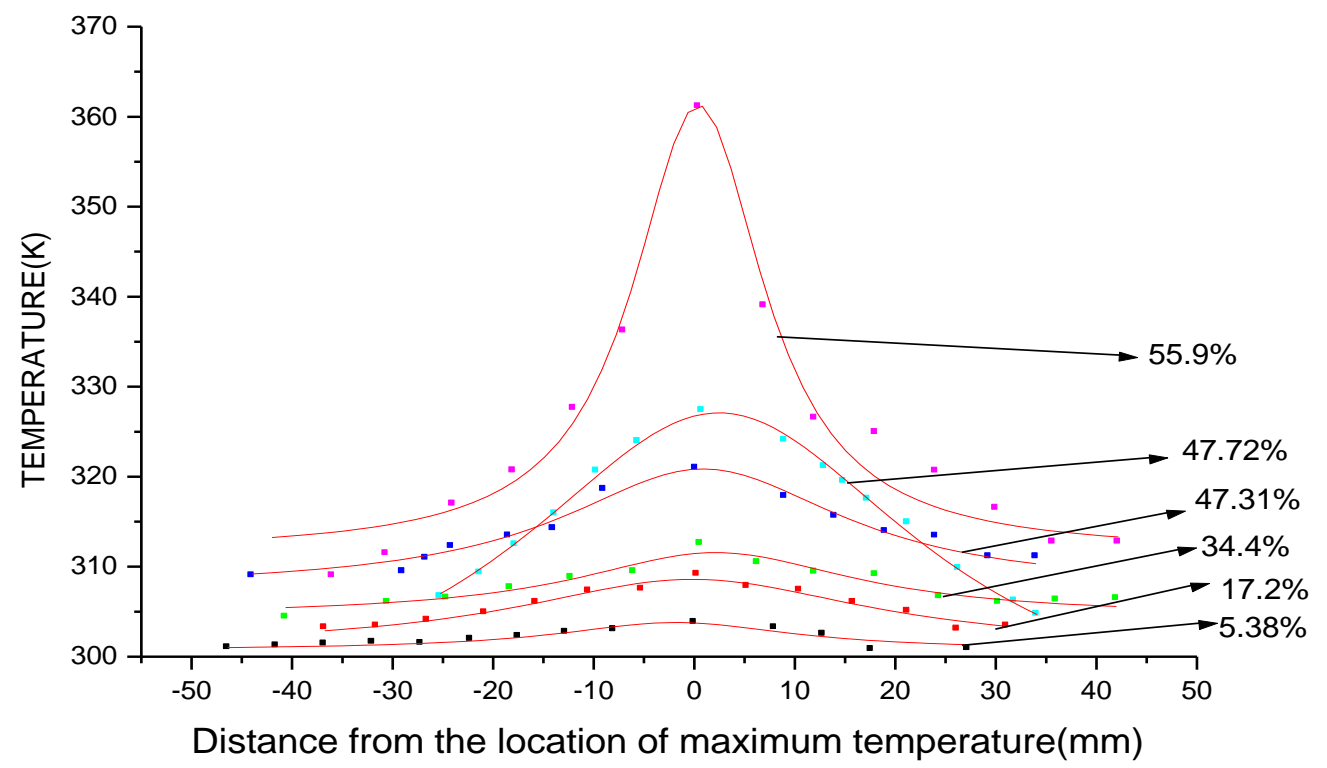

(b) Strain rate of $1.7 \times 10^{-2} \mathrm{~s}^{-1}$

Fig. 3. Temperature profile along gauge length for different strain levels

The data in Figs. 3. (a)-(b) have been fitted using the Lorentz equation

$$
Y=Y_{0}+\frac{2 A}{\pi} \frac{W}{4\left(x-x_{c}\right)+W^{2}}
$$

The results can be correlated with the mechanism of fracture in ductile metals.

The onset of necking is clearly revealed in thermography by the preferential heating that occurs with the temperature being maximum in the region of highest stress concentration and falling of drastically on 
either side of the centre. This is evident in Figs 3. (a)-(b) as well. Thus the zone of failure becomes very prominent, and it can be thus concluded that the process of necking elongation and the fracture of remaining ligaments is unambiguously revealed by thermography.

In earlier works on study of deformation induced heating, Ayres [42], Raghavan and Wagoner [43], had used thermocouples welded on to the specimen surface at suitable distances to measure temperature. However, such measurements gave only point information. Moreover, the errors associated with these measurements were found to be quite high $(> \pm 1 \mathrm{~K})$ since the exact point at which the maximum temperature occurs were not known. The present experiments clearly indicate that IR imaging provides a full-field thermal image obtained in a non-contact way from which accurate surface temperature measurements can be made. The full-field temperature image makes it possible to locate the point and time of strain localization during a dynamic test. This clearly proves that the technique of infrared imaging can identify and delineate the zone of crack growth and failure during tensile deformation.

\subsection{Application of MLP- ANN for temperature prediction}

A novel and first of its kind approach is the use of MLP-ANN for predicting the temperature and strain rate during tensile deformation of nuclear grade AISI type 316 SS. The experimental data that has been generated was first used for training the neural networks and then applied for prediction purposes.

While a number of network architectures such as Kohenon, Binary Hopfield etc., are available, the multilayered perceptron (MLP) was chosen since this is one of the most versatile artificial neural networks and is popular for data classification and prediction applications. The basic architecture of the MLP neural network is shown in Fig. 4.

\section{INPUT LAYER $\quad$ OUTPUT LAYER}

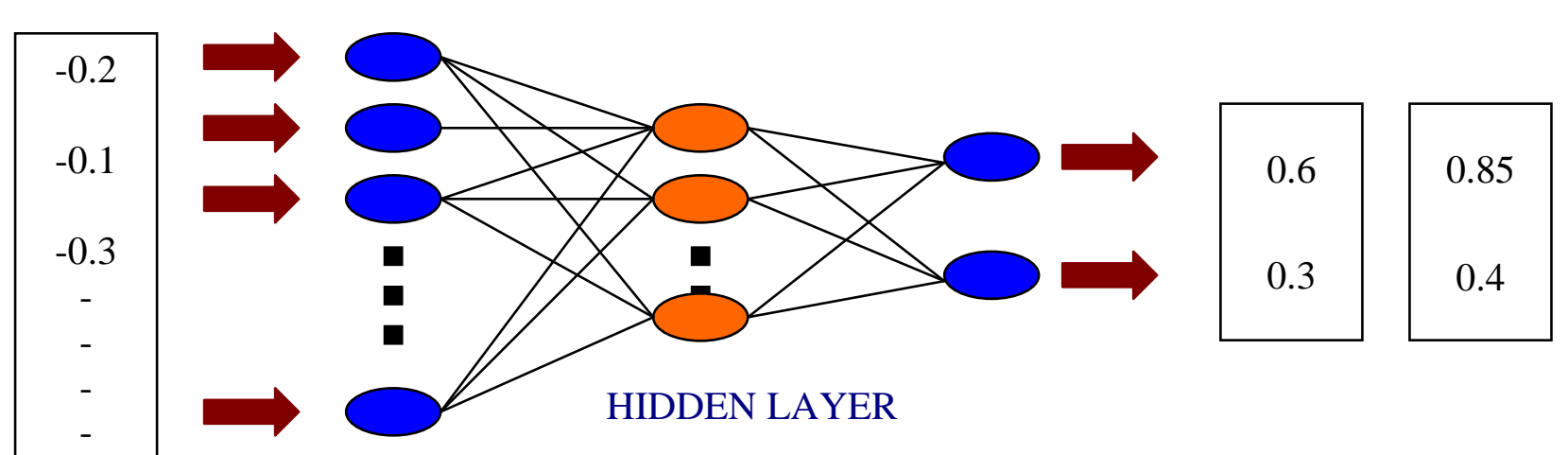

Input

Output

Pattern

Fig. 4. Basic Architecture of Multilayered Perceptron based Artificial Neural Network

The first layer, known as the input layer, consists of a number of input nodes. In general, there will be one such node for each variable used to model the output. In this case, it corresponds to the IR parameter (feature) used for classification/ prediction. Let $M$ represent the maximum number of variables (components per input vector) so that there would be $\mathrm{i}=1 \ldots \mathrm{M}$ nodes in the input layer. Each input vector $X_{i}$, then has $k=1 \ldots m$ values associated with it. The network contains one or more hidden layers and each node in the hidden layer is connected to every node in the previous layer through a set of weighted links. Let $\mathrm{j}$ represent the number of nodes in a hidden layer with $\mathrm{j}=1$...P such hidden layer nodes. Then there would be $\mathrm{M} \times \mathrm{P}$ such weights $\mathrm{W}_{\mathrm{ij}}$, connecting the input node to the hidden layer nodes. MLPs can have more than one hidden layer also. Following the hidden layers, is the output layer, which may contain a node for every variable that is to be modelled. Again, weights are used to connect each output node to 
every node in the previous hidden layer. With $\mathrm{N}$ output nodes and one hidden layer, there will be $\mathrm{N} \times \mathrm{P}$ such weights $\mathrm{w}_{\mathrm{jf}}$, connecting the hidden layer nodes to the output nodes.

The MLP can be viewed as a network whose weights are used to map a series of input patterns (or feature vectors) into the required output. Final values of all the link weights are obtained by a training process. The training is carried out by passing a set of inputs (in our case of IR study, these could be nominal strain, temperature, strain rate, cross head speed and stress.) through the MLP and adjusting the weights to minimize the error between the result the network gives for output, $Y_{\mathrm{fk}}^{\mathrm{p}}$ and the actual output value, $\mathrm{Y}_{\mathrm{fk}}$. This is repeated for all the input combinations and the final weights are those that minimize the average of all $n$ squared errors. Once the weights have been set, the network is able to produce predictions for the output using input values not used during the training process.

Fig. 5 shows the training process in more detail by illustrating the inputs between four input nodes $(i=1$ to 4) and a single node (node $j$ ) in the hidden layer.

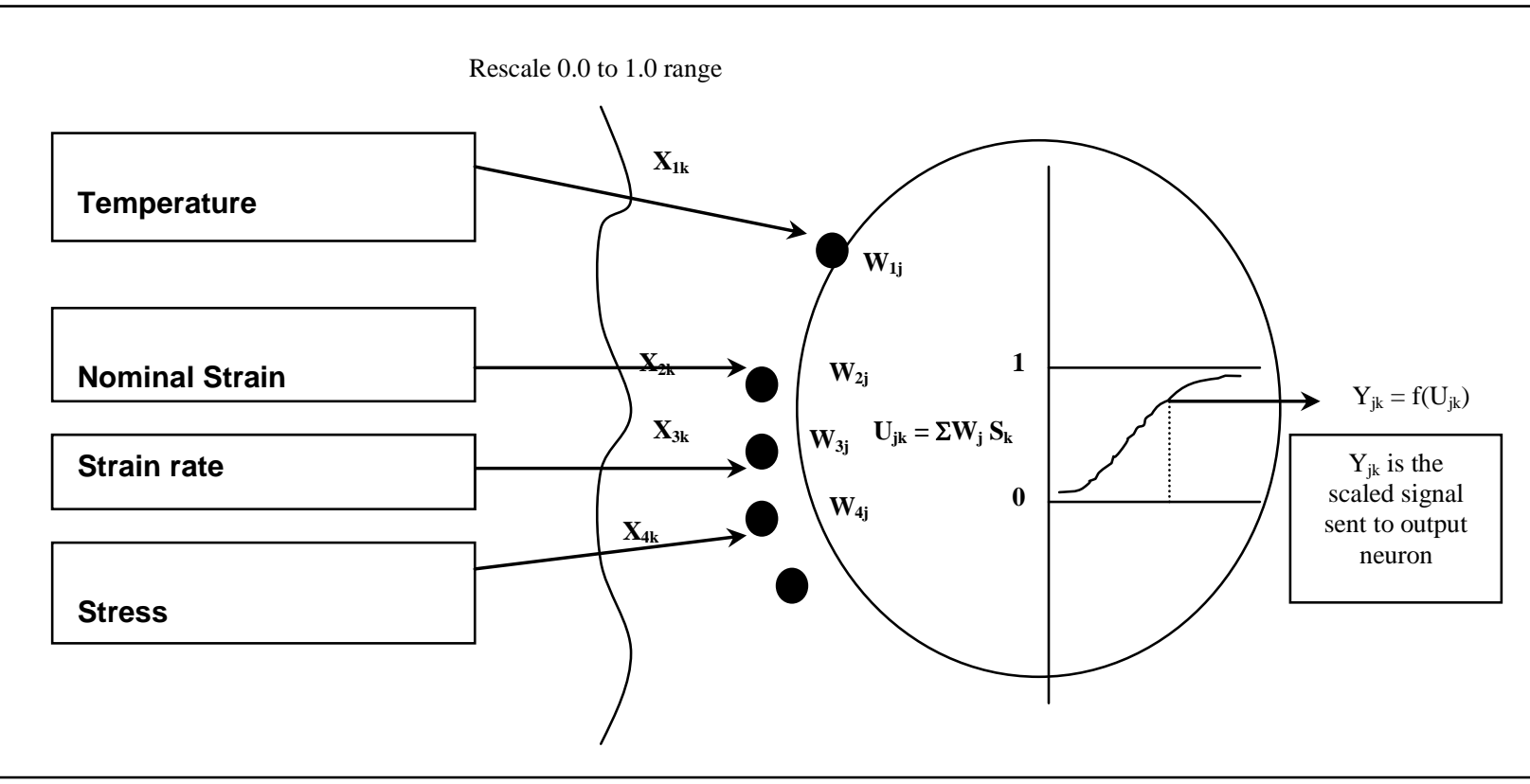

Fia. 5. Feed forward procedure throuah a part of the network

A set of input values $X_{1 k}$ to $X_{4 k}$ (where $X_{1 k}$ to $X_{4 k}$ might be temperature / nominal strain/ strain rate/stress obtained during tensile deformation (for example)) is presented to the network and a weighted sum of these values is formed using the relevant weights $\left(w_{1 j}\right.$ to $\left.w_{4 j}\right)$. This weighted sum $U_{j k}$ is then passed through a non- linear activation function $f\left(U_{j k}\right)$ to produce an output $Y_{j k}$ in the range 0 to 1 . It is because of this activation function that the MLP is ideal for modelling non-linear relationships such as that between temperature generated and strain or strain rate. This output is then sent to a node in the next hidden layer or to an output node where a similar weighting and normalization procedure takes place. The result will be an output prediction in the range 0 to 1 . To allow comparison to be made, the output data must be rescaled in this range before analysis. It is also advisable to rescale all inputs into a similar range so that the weighted sums described above are often calculated on the scaled inputs. For the following neural network prediction study, the DESKPACK Software System (DSS) was used. The parameters used in this study are (a) stress, (b) nominal strain, (c) maximum temperature, (d) strain rate corresponding to cross head velocity of $1 \mathrm{~mm} / \mathrm{min}, 2 \mathrm{~mm} / \mathrm{min}, 5 \mathrm{~mm} / \mathrm{min}, 10 \mathrm{~mm} / \mathrm{min}$ and $50 \mathrm{~mm} / \mathrm{min}$. The MLP-ANN is trained to predict temperature, and strain rate while feeding the other parameters as inputs. In this paper, we focus only on the temperature prediction.

The temperature is predicted with the input parameters being stress, nominal strain and strain rate. A total of 204 data vectors were used. The data vectors, were having wide ranging values. The values of temperature ranged from $273 \mathrm{~K}-373 \mathrm{~K}$, In order to use these values in the MLP-ANN effectively, these were mapped onto a scale between 0.1 and 0.9 , so as to increase the efficiency of the MLP-ANN. While 
mapping these values, the scaling factor was also saved, so that a reverse mapping could be done later to compare the original (strain rate or temperature) values with the predicted values. The 204 forwardmapped data vectors were divided into two sets (set1 and set2), each consisting of 102 vectors, using the DSS. After obtaining the two sets of input vectors viz., set1 and set2, in trial 1, the first set (set1) is used for training the MLP-ANN and the second set (set2) is used for testing (predicting). In trial 2, the second set (set2) is used for training the MLP-ANN and the first set (set1) is used for testing (predicting).

During the temperature prediction study, for both trial 1 and trial 2, the MLP-ANN architecture used is 4-32-1, i.e., the MLP-ANN had 4 nodes in the input layer, 3 nodes and 2 nodes in the hidden layer and one node in the output layer. This architecture achieved the minimum pattern error in this study. The architectural details of this MLP-ANN is given in Table 2.

Table 2. Architectural Details of MLP - ANN

\begin{tabular}{|l|l|}
\hline Input Nodes & 4 \\
\hline Hidden Layer & 2 \\
\hline Output Node & 1 \\
\hline Nodes in Hidden Layer 1 & 3 \\
\hline Nodes in Hidden Layer 2 & 2 \\
Nodes in Hidden Layer 3 & 0 \\
\hline Nodes in Hidden Layer 4 & 0 \\
\hline Nodes in Hidden Layer 5 & 0 \\
\hline Nodes in Hidden Layer 6 & 0 \\
\hline D.C.Bias Removal & No \\
\hline Normalisation & No \\
\hline Total Number of Files & 102 \\
\hline Record Length of Each Signal & 4 \\
\hline Mode (Train / Test) & Train \\
\hline Learning Rate & 0.700000 (Decreases with Epoch) \\
\hline Completed Max Epochs. & 75,000 \\
\hline Final Cumulative Pattern Error & 0.049608 \\
\hline
\end{tabular}

A final pattern error of 0.049608 was achieved as indicated in Table 2. It took the MLP-ANN, 75000 epochs to achieve this error reduction.

Once the MLP-ANN is trained with set1 and the lowest pattern error is achieved, the second set (set2), was used as a testing set and the corresponding temperature values for a strain rate of $1.7 \times 10^{-4} \mathrm{~s}^{-1}$ were predicted. Fig. 6 is the plot of the experimental and theoretical predictions with respect to nominal strain for a strain rate of $1.7 \times 10^{-4} \mathrm{~s}^{-1}$. The temperature values obtained from MLP-ANN matched with the experimentally observed values. The overall error was less than $1 \%$.

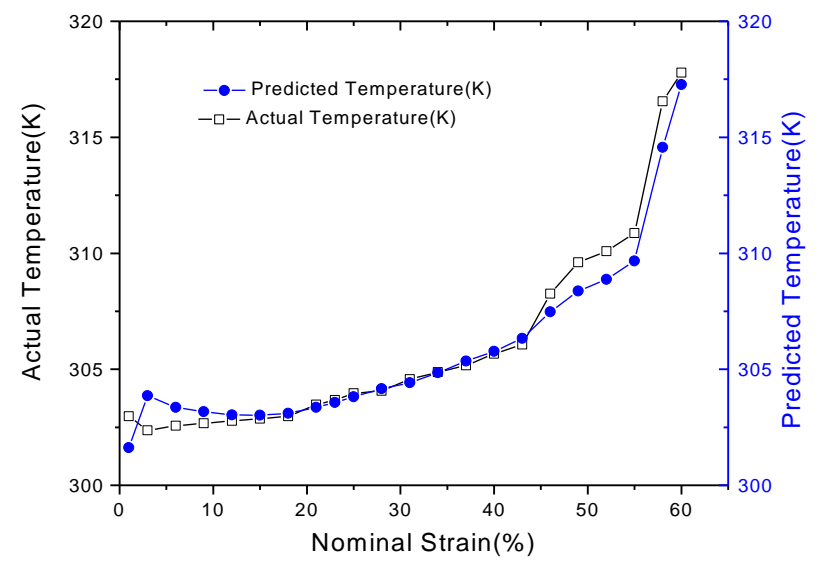

Figure 6. Plot of actual and predicted temperatures for a strain rate of $1.7 \times 10^{-4} \mathrm{~s}^{-1}$. 


\subsubsection{Error in Temperature Prediction}

A statistical analysis of the actual and predicted temperature values was also carried out. If $\bar{T}$ is the mean value of the temperature, then the standard deviation $(S D)$ is given by :

$$
S D \equiv \sqrt{\operatorname{Var}} \equiv \frac{1}{n-1} \sum_{i=1}^{n}\left(T_{i}-\bar{T}\right)^{2}
$$

where $\operatorname{Var}$ is the variance and $n$, the sample size. The standard error of the mean ( SEM ) is given by

$$
S E M \equiv \frac{S D}{\sqrt{n}}
$$

The result of this analysis is given in Table 3 for the typical case of strain rate of $1.7 \times 10^{-4} \mathrm{~s}^{-1}$.

Table 3. Statistical Analysis of Actual and Predicted Temperatures for a Strain Rate- $1.7 \times 10^{-4} \mathrm{~s}^{-1}$

\begin{tabular}{|c|c|c|c|c|}
\hline $\begin{array}{l}\text { Actual Temperature } \\
(\mathbf{K})\end{array}$ & $\begin{array}{l}\text { Predicted } \\
\text { Temperature(K) }\end{array}$ & $\begin{array}{l}\text { Mean } \\
(\mathbf{K})\end{array}$ & $\begin{array}{l}\text { Standard } \\
\text { Deviation (K) }\end{array}$ & Error (\%) \\
\hline 302.9699 & 301.6268 & 302.2983 & 0.9497 & 0.67154 \\
\hline 302.3701 & 303.8598 & 303.1149 & 1.05339 & 0.74486 \\
\hline 302.5701 & 303.3536 & 302.9619 & 0.554 & 0.39174 \\
\hline 302.6697 & 303.1763 & 302.923 & 0.35824 & 0.25331 \\
\hline 302.7698 & 303.0343 & 302.902 & 0.18703 & 0.13225 \\
\hline 302.8698 & 303.0169 & 302.9434 & 0.10401 & 0.07355 \\
\hline 302.9699 & 303.1046 & 303.0372 & 0.09529 & 0.06738 \\
\hline 303.4696 & 303.3623 & 303.416 & 0.07592 & 0.05368 \\
\hline 303.6697 & 303.5724 & 303.6211 & 0.06882 & 0.04866 \\
\hline 303.9699 & 303.8127 & 303.8913 & 0.11111 & 0.07857 \\
\hline 304.0699 & 304.159 & 304.1145 & 0.06299 & 0.04454 \\
\hline 304.5697 & 304.4194 & 304.4945 & 0.10628 & 0.07515 \\
\hline 304.8698 & 304.8538 & 304.8618 & 0.01131 & 0.008 \\
\hline 305.1695 & 305.3513 & 305.2604 & 0.12857 & 0.09091 \\
\hline 305.6697 & 305.7748 & 305.7222 & 0.0743 & 0.05254 \\
\hline 306.0694 & 306.3303 & 306.1999 & 0.18446 & 0.13043 \\
\hline 307.4696 & 308.2567 & 307.8632 & 0.55657 & 0.39355 \\
\hline 308.3691 & 309.6126 & 308.9909 & 0.87929 & 0.62175 \\
\hline 308.8694 & 310.085 & 309.4772 & 0.85959 & 0.60782 \\
\hline 309.6693 & 310.8694 & 310.2693 & 0.84858 & 0.60004 \\
\hline 314.5688 & 316.5464 & 315.5576 & 1.39837 & 0.9888 \\
\hline 317.2687 & 317.7862 & 317.5274 & 0.36596 & 0.25877 \\
\hline & & & & \\
\hline & & & & \\
\hline
\end{tabular}

It can be observed from figure 6 and also from Table 3 that the error between the actual and predicted temperatures is high in the initial elastic region, being of the order of $0.6-0.7 \%$, and towards the end after the ultimate tensile strength $(0.6-0.98 \%)$ while in the intermediate regions there is excellent correlation, with the error being less than $0.1 \%$. This can be explained based on figure 2 .

Three distinct regions can be visualised in figure 2 - the first being from start of the test to just before yield point (nominal strain of about 8-10\%), the second region from yield point to UTS (nominal strain of about $10 \%-60 \%)$ and the third region from UTS to fracture. The first region indicates an initial rise followed by a small drop in the temperature attributed to the thermoelastic effect. The second region is the region of work hardening in which the temperature rises gradually with strain. In the third region immediately after UTS, there is a sudden and drastic rise in the temperature. The ANN is modelled on the overall basis of increasing values of temperature with increasing stress and strain rates using a sigmoidal function. Thus, 
we have excellent correlation in the work hardening region where the temperature increases gradually with strain, while in regions I and III, due to the non-linear variation of temperature with nominal strain, the error is quite high. Hence the ANN model predicts a temperature value, which is greater by about $0.2 \mathrm{~K}$ to $0.6 \mathrm{~K}$ compared to the actual values. However, it can be observed from Table 2 that the error in prediction of temperature values does not exceed 0.98 , in general, with excellent correlation in the work hardening region (errors $<0.1 \%$ ).

\section{Conclusion}

The results presented in this thesis represent a successful experimental attempt to characterise the thermal signature generated during the entire process of tensile deformation of AISI type 316 SS upto fracture as a function of nominal strain and strain rate. All the stages of tensile deformation i.e., "microyielding, yielding, progressive plastic deformation and necking elongation and fracture" could be identified and characterized by IR imaging. The results also clearly reveal that IR imaging (thermography/thermal imaging) is quite sensitive and complements mechanical testing by providing online visualisation and with the full-field thermal image from which accurate surface temperature measurements, without contacting the specimen, are possible. The application of full-field temperature imaging makes it possible to locate the point and time of strain localization during a tensile test. Profiling of the temperature along the gage length clearly proves that the technique of infrared imaging can identify and delineate, well in advance, the zone of failure during tensile deformation. This result could also be utilized in practical situations to identify possible highly stressed zones in component before component fails.

A novel approach is the use of MLP-ANN for predicting the temperature and strain rate during tensile deformation of nuclear grade AISI type 316 SS. The experimental data that have been generated were first used for training the neural networks and then for prediction purposes. In the case of temperature prediction, the overall error is less than $1.0 \%$. The percentage errors can be further minimised by

1. Having more number of samples for training the network

2. Training the network to predict the temperature zone / region wise - before yield, work hardeniniy phase and beyond UTS. This should improve the accuracy of prediction considerably.

\section{Acknowledgements}

The authors are thankful to Dr P.R.Vasudeva Rao, Director, IGCAR and Shri S.A.V.Satya Murty for their encouragement and support. Authors are also thankful to $\mathrm{Dr}$ C.K.Mukhophadyay, NDED, IGCAR for his experimental support in tensile testing and Dr. C.Rajagopalan formerly at DPEND, IGCAR Kalpakkam for the DESK PACK System developed by him and for MLP-ANN analysis.

\section{References}

[1] David K. Wilburn, Temperature profiles observed in tensile specimens during physical test, Materials Evaluation, 1977, 35(3), 28-31.

[2] Y.Huang, J.Xu and C.H. Shih, Application of Infrared Technique to research on Tensile Test , Materials Evaluation, $1980,12,76-79$.

[3] Anil K.Sachdev and Joseph E.Hunter Jr., Thermal Effects during Uniaxial straining of steels, Metallurgical Transactions A, 1982, 13A, 1063.

[4] B.Venkatraman, Infrared imaging for characterisation of weld defects, crack growth and plastic deformation.

[5] Robert A.Ayres, Thermal Gradients, Strain Rate, Ductility in Sheet Steel Tensile Specimens, Metallurgical Transactions A, 16A, 1985, pp.37-43.

[6] K.S.Raghavan and R.H.Wagoner, International Journal of Plasticity, 3, 1987, pp. 33.

[7] B. Venkataraman, C. K. Mukhopadhyay and Baldev Raj, Early Prediction of the Failure Zone during Tensile Deformation of AISI type 316 Stainless Steel using Infrared Thermography, Experimental Techniques, Vol. 28, No. 2, pp. 35-38. 